\title{
Successful Prolonged Use of Ketamine Intravenous Infusion in Status Asthmaticus
}

\author{
Muhammad H Gul ${ }^{1 *}$, Suresh Kumar ${ }^{1}$ and Zin Mar Htun ${ }^{2}$ \\ ${ }^{1}$ Department of Internal Medicine, Amita-Presence Saint Joseph Hospital, USA \\ ${ }^{2}$ Department of Internal Medicine, Louis A Weiss Memorial Hospital, USA
}

*Corresponding author: Muhammad H Gul, Amita-Presence Saint Joseph Hospital, Chicago,

\section{Case Report}

Volume 5 Issue 1

Received Date: January 27, 2020

Published Date: February 14, 2020

2900 N Lakeshore Drive, Chicago, IL, 60657, USA, Tel: 407-443-6688; Email: hamdan3802@hotmail.com

\section{Abstract}

Status asthmaticus is a life-threatening condition with significant morbidity and mortality. There is limited experience with the use of ketamine intravenous infusion as a sedative in mechanically ventilated status asthmaticus patients. We are reporting a case of mechanically ventilated status asthmaticus patient being successfully managed with prolonged use of ketamine infusion. A 20-year-old female with a history of asthma was admitted for acute severe asthma exacerbation. She had worsening acute hypercarbic respiratory failure despite the treatment with a traditional bronchodilator, steroids, and magnesium, and she was intubated. She became hypotensive with the use of propofol and was started on ketamine infusion, which was continued for four days. Ketamine provided better sedation, blood pressure stability, and gradual improvement in bronchospasm, which led to the successful extubation and resolution of the asthma attack. She did not report any nightmares or dreams with the use of ketamine. Prolonged use of ketamine infusion has not been studied in acute asthma exacerbation. We report a case where ketamine infusion was successfully used for four days in an intubated patient with status asthmaticus without any significant side effects.

Keywords: Ketamine; Asthmaticus; Cisatracurium; Extubation

\section{Introduction}

Status asthmaticus is an acute exacerbation of asthma that remains unresponsive to initial treatment with bronchodilators. Status asthmaticus can result in hypoxemia, hypercarbia, and secondary respiratory failure. It is associated with significant mortality, particularly in patients when medical treatment is delayed. Ketamine is a sedative analgesic with bronchodilator properties, which has been used with limited experience in asthma patients [1]. We present a case of severe asthma exacerbation in an adult female patient where intravenous (IV) ketamine was successfully used in a ventilated patient in addition to the standard treatment of asthma.

\section{Case Description}

A 20 year-old-female with a past medical history of moderate persistent asthma since childhood presented to the emergency room with shortness of breath, which woke her up from sleep early in the morning. It was associated with wheezing and chest tightness and not relieved by an albuterol inhaler. On examination, she was in respiratory distress, using accessory muscles, and unable to complete sentences. Vitals signs were stable except for tachypnea with respiratory rate 25 breaths/min and tachycardia with heart rate 125 beats/min and oxygen saturation $100 \%$ at room air. Lungs examination was notable for diffuse wheezing. Labs revealed normal complete blood cell count and serum 


\section{Open Access Journal of Pulmonary \& Respiratory Sciences}

creatinine. Chest X-ray demonstrated radiographically clear lungs. The patient received albuterol nebulization and oral prednisone, subcutaneous epinephrine, and magnesium sulfate, and was placed on bi-level positive airway pressure (BiPAP) due to respiratory distress. The initial set of arterial blood gas (ABG) was done right after the placement of BiPAP, which revealed acute hypercapnia. The patient continued to be in respiratory distress with diaphoresis, severe tachypnea, and the decision was made for mechanical ventilation with endotracheal intubation. On the ventilator, the patient was noted to have breath-stacking with peak airway pressure as high as $90 \mathrm{~cm} \mathrm{H2O}$. The patient was sedated with increasing doses of intravenous lorazepam, propofol, and paralyzed with cisatracurium infusion. However, the patient developed hypotension resistant to intravenous fluid boluses, requiring intravenous epinephrine push. The ventilator was disconnected to allow the exhalation of trapped air. The ventilator settings were adjusted to decrease the intrinsic positive end-expiratory pressure (auto-PEEP). Propofol was discontinued due to hypotension, and she was placed on fentanyl, midazolam infusion and intravenous ketamine infusion at $1 \mathrm{mg} / \mathrm{kg} / \mathrm{hr}$. The patient was started on intravenous methylprednisone 60 milligrams every 8 hours, and frequent bronchodilator treatments. Her blood pressure stabilized, and the peak pressures improved in the following days. Cisatracurium was continued for four days due to persistently elevated peak pressure noticed when cisatracurium was weaned off. After four days of admission, wheezing had improved, ketamine and cisatracurium were discontinued, and the patient was successfully extubated on the sixth day of admission. She did not report any hallucinations, nightmares, or dreams after being extubated. She displayed a rational thought process and appropriate behavior. She was discharged home on weaning doses of oral prednisone.

\section{Discussion}

Status asthmaticus is considered a medical emergency that is associated with significant mortality and cost. This case demonstrates the successful use of ketamine infusion for four days in a patient with severe status asthmaticus without any clinically significant side effects. Ketamine is a well-known drug with safe and predictable sedative, analgesic, and antiemetic effects. It is also a known bronchodilator [2] and has been used as an inductive agent in the rapid sequence intubation of asthma patients ${ }^{3}$. The mechanism of ketamine-induced bronchodilation includes diminishing the vagally induced airway constriction [3], stimulating the release of catecholamines [4], blocking of the NMDA receptors [5], and the direct relaxant effect on airway smooth muscle [3]. Although experience with this therapy is limited, several case reports have described the successful treatment of children and adults with status asthmaticus with intravenous ketamine. Low dose ketamine infusion $0.4-0.5 \mathrm{mg} / \mathrm{kg}$ has been found efficacious in the emergency department for the treatment of acute asthma attack [6]. A prospective observational study demonstrated the efficacy of short term use of ketamine-limited to two hours-in cases of status asthmaticus admitted to the intensive care unit [6]. However, there is limited experience with the prolonged use of ketamine infusion in asthma patients. To our knowledge, Park et al. reported the only other case where ketamine was used in asthma patients for five days, where it helped with the resolution of asthma; however, the patient was noted to have subsequent frustration and feelings of guilt about racist dreams [7]. In other studies, the use of ketamine was mostly limited to three days [1]. Ketamine has a dissociative anesthetic effect and has the potential to be abused [8]. Chronic use of ketamine has been associated with cognitive impairment and addiction [9]. Clinicians should be aware of its psychiatric side effects, including confusion, delirium, agitation, and hallucinations $[10,11]$. The patient was not reported to have any of these side effects on extubation in our case. Ketamine is also known to increase blood pressure [12], which makes it a feasible choice of sedation in hypotensive asthmatic patients in contrast to propofol. Our case demonstrates the successful prolonged use of ketamine without any significant side effects in a ventilated patient with asthma.

\section{References}

1. Goyal S, Agrawal A (2013) Ketamine in status asthmaticus: A review. Indian J Crit Care Med 17(3): 154161.

2. Vitkun SA, Michael Foster W, Chang H, Bergofsky EH, Poppers PJ (1987) Bronchodilating effects of the anesthetic ketamine in an in vitro guinea pig preparation. Lung 165(1): 101-113.

3. Brown Robert H, Wagner Elizabeth M (1999) Mechanisms of Bronchoprotection by Anesthetic Induction Agents: Propofol versus Ketamine. Anesthesiology: The Journal of the American Society of Anesthesiologists 90(3): 822828.

4. Hanouz JL, Zhu L, Persehaye E, Massetti M, Babatasi G (2005) Ketamine Preconditions Isolated Human Right Atrial Myocardium: Roles of Adenosine Triphosphatesensitive Potassium Channels and Adrenoceptors. Anesthesiology: The Journal of the American Society of Anesthesiologists 102(6): 1190-1196.

5. Sato T, Hirota K, Matsuki A, Zsigmond EK, Rabito SF (1998) The role of the N-methyl-D-aspartic acid receptor in the relaxant effect of ketamine on tracheal smooth muscle. Anesth Analg 87(6): 1383-1388. 


\section{Open Access Journal of Pulmonary \& Respiratory Sciences}

6. Esmailian M, Koushkian Esfahani M, Heydari F (2018) The Effect of Low-Dose Ketamine in Treating Acute Asthma Attack; a Randomized Clinical Trial. Emerg (Tehran) 6(1): e21-e21.

7. Park GR, Manara AR, Mendel L, Bateman PE (1987) Ketamine infusion. Anaesthesia 42(9): 980-983.

8. Liu Y, Lin D, Wu B, Zhou W (2016) Ketamine abuse potential and use disorder. Brain Res Bull 126: 68-73.

9. Ding R, Li Y, Du A, Yu H, He B, et al. (2016) Changes in hippocampal AMPA receptors and cognitive impairments in chronic ketamine addiction models: another understanding of ketamine CNS toxicity. Scientific
Reports 6(1): 38771.

10. Rasmussen KG (2014) Psychiatric side effects of ketamine in hospitalized medical patients administered subanesthetic doses for pain control. Acta Neuropsychiatrica 26(4): 230-233.

11. Britt GC, McCance-Katz EF (2005) A Brief Overview of the Clinical Pharmacology of "Club Drugs". Substance Use \& Misuse 40(9-10): 1189-1201.

12. Suleiman Z, Ik K, Bo B (2012) Evaluation of the cardiovascular stimulation effects after induction of anaesthesia with ketamine. J West Afr Coll Surg 2(1): 3852. 